ISAHP 2007, Viña del Mar, Chile, August 8-3, 2007

\title{
AUTOMATIC BUILDING QUESTIONAIRES SOFTWARE FOR SUPERDECISIONS MODELS
}

\author{
Juan Pascual Pastor Ferrando, Pablo Aragonés Beltrán, \\ Mónica García Melón, Rocío Poveda Bautista \\ Universidad Politécnica de Valencia \\ Camino de vera s/n, 46022, Valencia (SPAIN) \\ jppastor@dpi.upv.es, aragones@dpi.upv.es,mgarciam@dpi.upv.es, ropobau@dpi.upv.es
}

\begin{abstract}
When elaborating models in ANP, there are two key activities in the process: identify the relationships and weight the relationships to obtain the unweighted and weighted supermatrixes. Habitually this information is avalaible from experts, to those that it is necessary to ask. So, is very interesting build appropriates questionnaires to extract the information that they posses. These questionnaires should be on printed paper for several reasons: the expert doesn't have time to respond in a work session and it should be facilitated so that he gives it to us once answered, to have a font of tracing the data, etc. In big network models, once identified the correct form of asking, elaborate the questionnaires manually is a heavy and arduous task that doesn't contribute value to the decisionmaking process.

In this article we show up an application that reads the files of the software Superdecisions, and it elaborates automatically the questionnaires to define the relationships and the questionnaires to prioritize the existing relationships.
\end{abstract}

Keywords:

ANP, SuperDecisions, Facilitator tools, Relationships questionnaires, Priorization questionnaires 




\section{Introduction}

When we are solving a problem of decision, the model construction for decision aid is a fundamental task. This activity is usually carried out conjointly among those responsible for the decision and the facilitator or consultant in multicriteria. Habitually the decisor has a comprehensive knowledge of the problem and he senses which are the most important aspects, while the facilitator contributes to extract this information and to organize it through different questions, during several work sessions. Elaborating ANP model, this process could be summarized in a series of iterations of the following steps:

- $\quad$ Identification of the elements that intervene in the decision.

- $\quad$ Grouping or classification of these elements according to common aspects.

Once identified the elements and the groups, the following phase is to identify the network topology that better it adapts to the pattern. Basically, could be defined two opposed typologies: an only network with all the groups in the same level, or a network structured in different levels. To choose the form of the network depends in great measure of the relationships that are defined in the decision aid model. To identify these relationships it is fundamental to carry out all the possible questions to the decisor. This process bring up a first great difficulty: the high number of possible relationships and, therefore, of questions. In a model with 20 elements, they are necessary 400 questions allowing reflexive influences, or 380 without allowing these. Another possible difficulty resides in the procedure to obtain the information of the existence of these relationships. With SuperDecisions software these relationships can be inserted in the model in a quick and friendly way, but, how to be safe that any relationships from and toward a certain cluster has not forgotten? How can you be safe that a relationship has not been inserted by mistake? This drawback is aggravated by the low people's available with the knowledge to appraise the relationships. It is very practical to give a questionnaire on paper so that the expert can respond to the questions according to his available fund of time, and with the record written it has responded on what.

Build these questionnaires in a manual way is a tedious task, and the dedicated time has to write them it doesn't contribute to add great value to the decision-making process. On the other hand, it contributes the drawback of carrying out these questionnaires erroneously: to forget some questions, to repeat other, etc.

A similar status takes place when already prioritizing the relationships defined to obtain the non weighted supermatrix, the weighted clusters matrix and the weighted supermatrix.

A software is showed in this paper, SuperDecisons-Q, to generate these questionnaires starting from the defined model by means of the software SuperDecisions. The software has been implemented in language JAVA.

\section{SuperDecisions-Q software}

The software development has tried to complete two opposed objectives: "simplicity and easy of using" versus "flexibility and adaptation to the desires of the possible users". This way, it has been opted for an easy and organized environment. The software comprises, basically, two windows:

- In the first one the file of SuperDecisions is loaded, and the questions of three questionnaires are defined: definition of relationships, comparison of clusters influences, comparison of influences among nodes. (Figure 1)

- In the second window the options of the software on the implementation of the questionnaires are defined. 


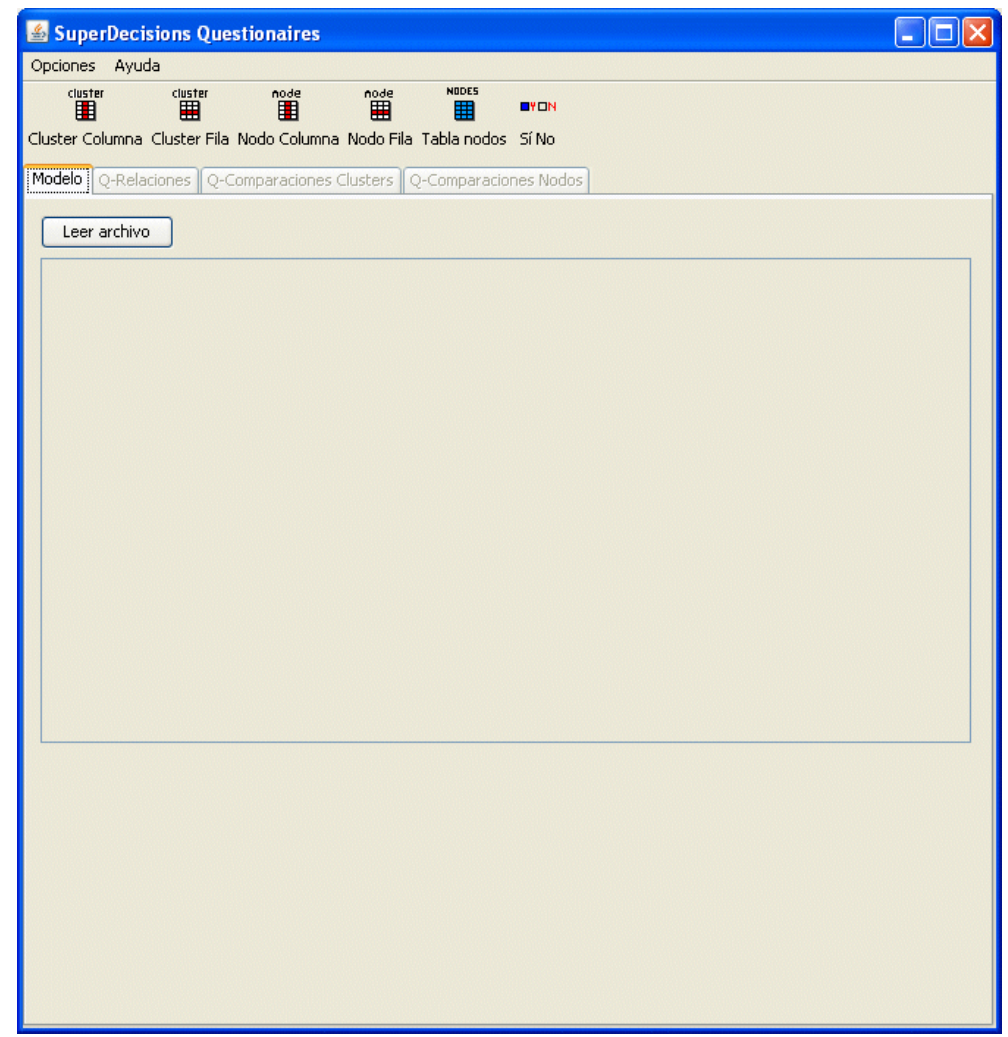

Figure 1.- Main screen

In the first window, the first step is to open the SuperDecisions file. Once loaded, the model is shown with the different subnetworks, if they exist. (Figure 2)

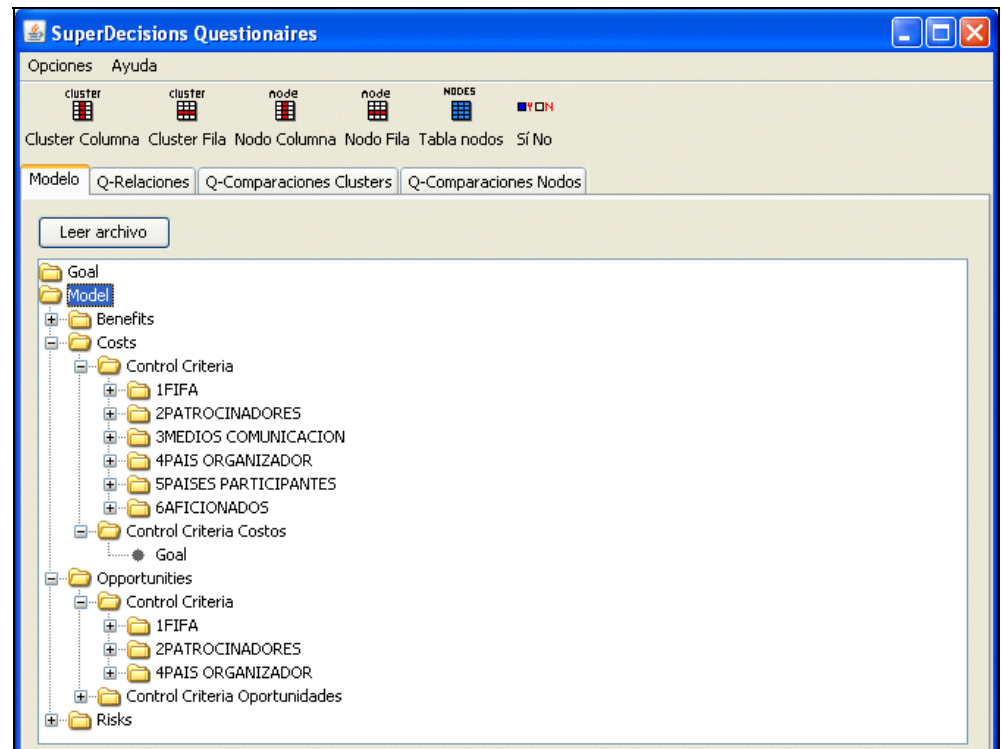

Figure 2.- Tabbed pane Model 


\subsection{Options}

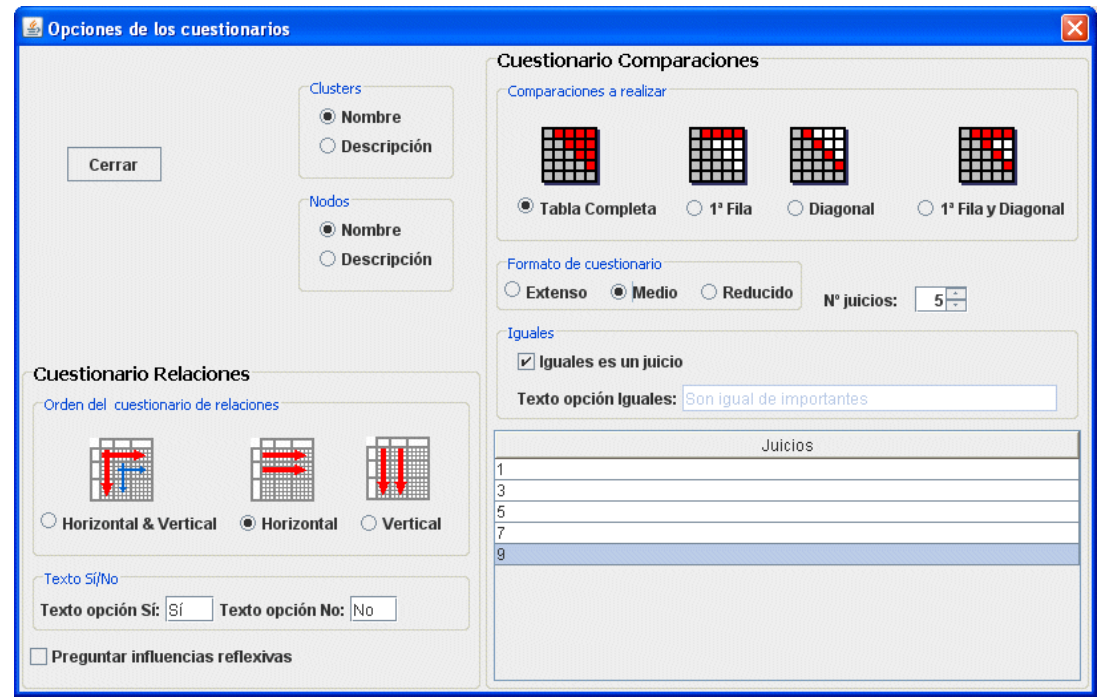

Figure 3.- Options dialog

The software has three groups of options: those that affect to the relationships questionnaires, those that affect to the questionnaires of comparisons, and other common to both.

1. In the first place we comment these last options. You can use the NAME or DESCRIPTION of the clusters or nodes.

2. The relationships questionnaire options allow to define the order of the questions, that is, like it is swept across the cells of the matrix of each network:

- $\quad$ VERTICAL: asking the influences on each one of the model's nodes,

- HORIZONTAL: asked on that it influences each one of model's nodes,

- HORIZONTAL \& VERTICAL: for each node, first the questions about on that others nodes influences each element, second to ask the influences on him.

- In the clusters questions with a single node, it is allowed to insert the equivalent text to YES and NOT in the language in the one that the questionnaire is generated.

- $\quad$ Lastly questions about reflexive relationships can be allowed.

3. As for the weighting questionnaire, one can choose the number of questions to carry out:

- $\quad$ All, to prioritize $n$ elements: $(\mathrm{n} \times(\mathrm{n}-1)) / 2$

- $\quad$ The minimum (n-1) corresponding to the first row of the pairwise matrix.

- The minimum (n-1) corresponding to the diagonal of the pairwise matrix of comparisons.

- $\quad$ Those corresponding to the first row and to the diagonal; $2 \mathrm{x} \mathrm{n}-3$.

4. The format of the comparisons questionnaires can be defined too. Three formats have been implemented that are shown next (Figures 3, 4 and 5). If the format allows it the user can write the judgments that are allowed.

\begin{tabular}{|l|l|l|l|l|}
\hline Element 1 & $\square$ & Element 2 & $\square$ & \\
\hline Element 1 & $\square$ & Element 3 & $\square$ & \\
\hline Element 1 & $\square$ & Element 4 & $\square$ & \\
\hline Element 2 & $\square$ & Element 3 & $\square$ & \\
\hline Element 2 & $\square$ & Element 4 & $\square$ & \\
\hline Element 3 & $\square$ & Element 4 & $\square$ & \\
\hline
\end{tabular}

Figure 4.- Short style 
A: Element 1

B: Element 2

\begin{tabular}{|l|l|l|l|l|l|}
\hline Text 1 & $\square$ A & $\square B$ & \multicolumn{3}{|l}{} \\
\cline { 1 - 3 } Text 2 & $\square 1$ & $\square 3$ & $\square 5$ & $\square 7$ & $\square 9$ \\
\hline
\end{tabular}

A: Element 1

B: Element 3

\begin{tabular}{|l|l|l|l|l|l|}
\hline Text 1 & $\square$ A & $\square B$ & \multicolumn{3}{l|}{} \\
\hline Text 2 & $\square 1$ & $\square 3$ & $\square 5$ & $\square 7$ & $\square 9$ \\
\hline
\end{tabular}

Figure 5.- Medium style

Comparation question

$\square$ Element 1

$\square$ Element 2

$\square$ Equals text

Text 1

\begin{tabular}{|l|l|}
\hline$\square$ & Judgement 1 \\
\hline$\square$ & Judgement 2 \\
\hline$\square$ & Judgement 3 \\
\hline$\square$ & Judgement 4 \\
\hline$\square$ & Judgement 5 \\
\hline
\end{tabular}

Comparation question

$\square$ Element 1

$\square$ Element 3

$\square$ Equals text

Text 1

\begin{tabular}{|l|l|}
\hline$\square$ & Judgement 1 \\
\hline$\square$ & Judgement 2 \\
\hline$\square$ & Judgement 3 \\
\hline$\square$ & Judgement 4 \\
\hline$\square$ & Judgement 5 \\
\hline
\end{tabular}

Figura 6.- Wide style

\subsection{Questionnaire tabbed panes}

In the relationships questionnaire tabbed pane (Figure 6) text questionnaire can be wrote:

- Cluster change text.

- $\quad$ Node change text.

- Question of clusters relationship.

- Question of relationship of nodes.

- Clusters question with a single node.

According to the active panel, it is allowed to introduce the references of clusters and nodes with the toolbar buttons.

In a similar way, the fields of comparations questionnaires are fulfilled in its tabbed panes. 


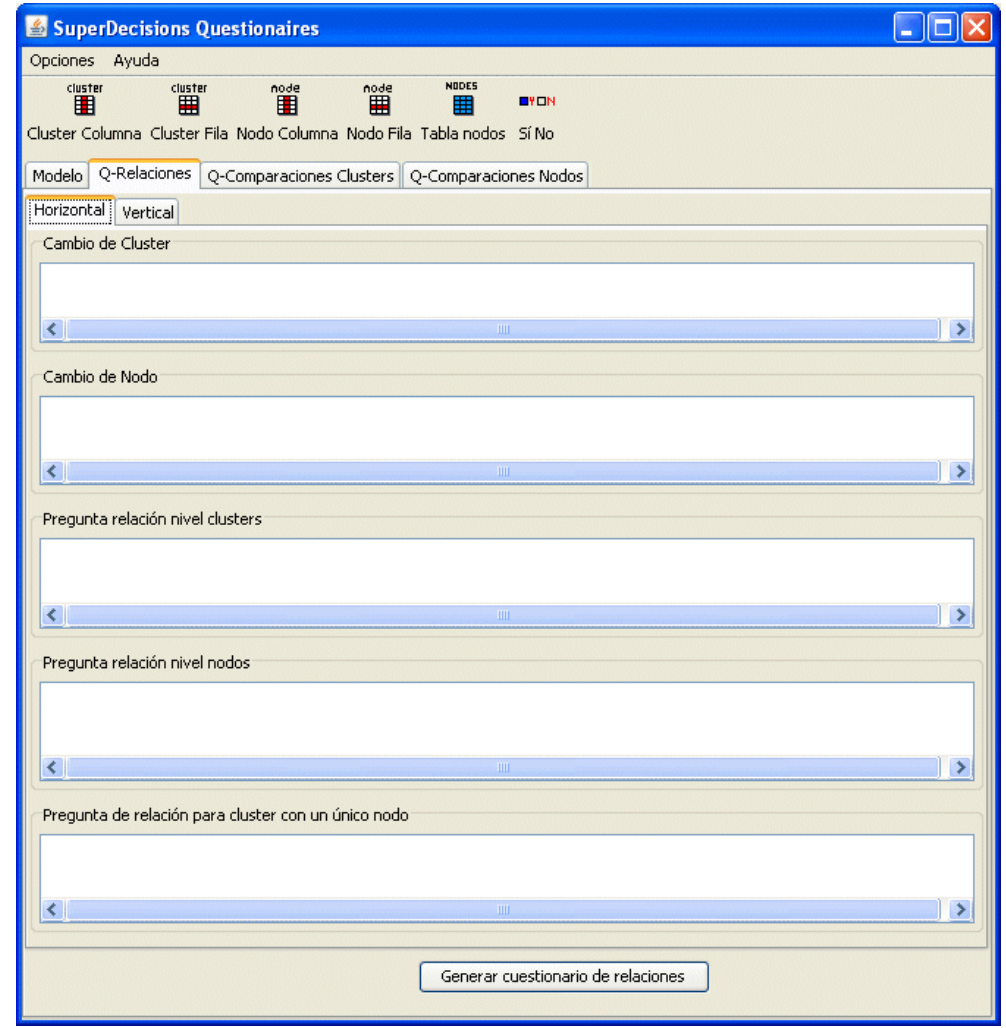

Figure 7.- Relationships questionnaire

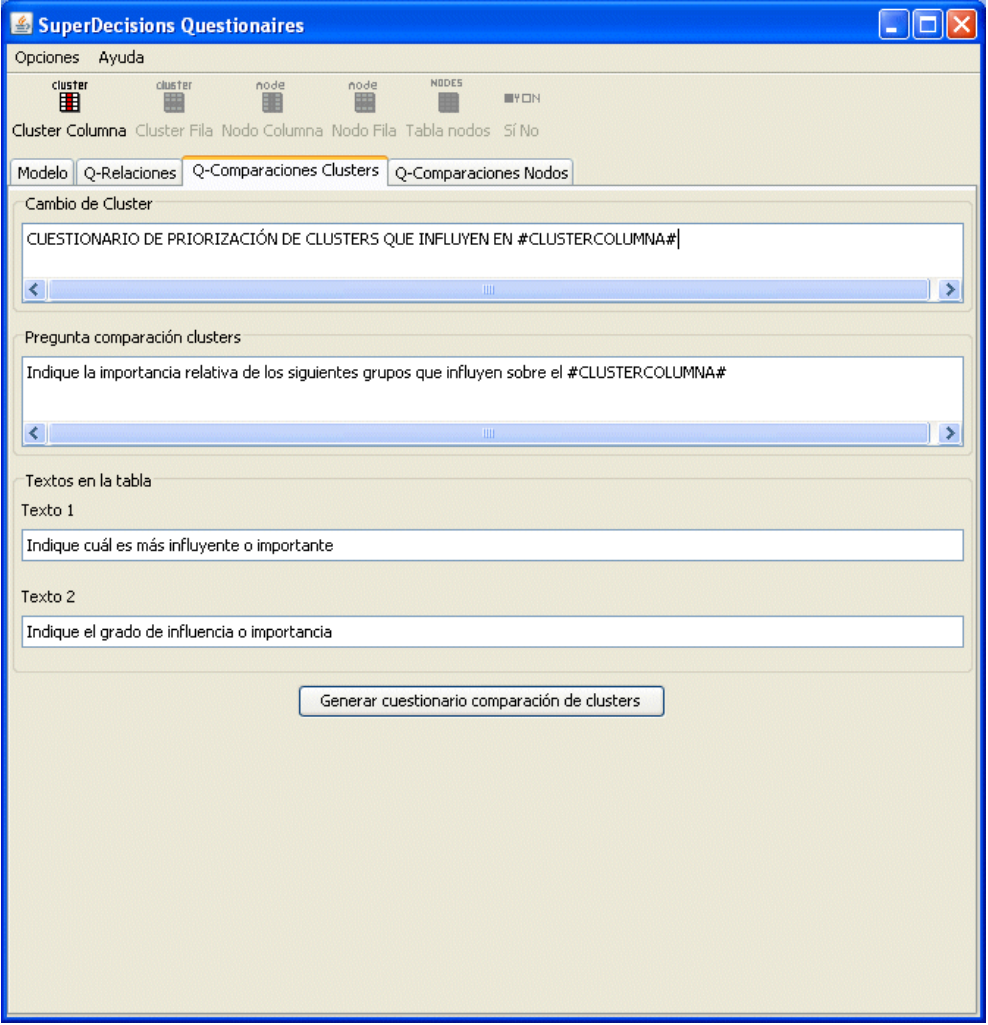

Figure 8.- Weighting clusters questionnaire 


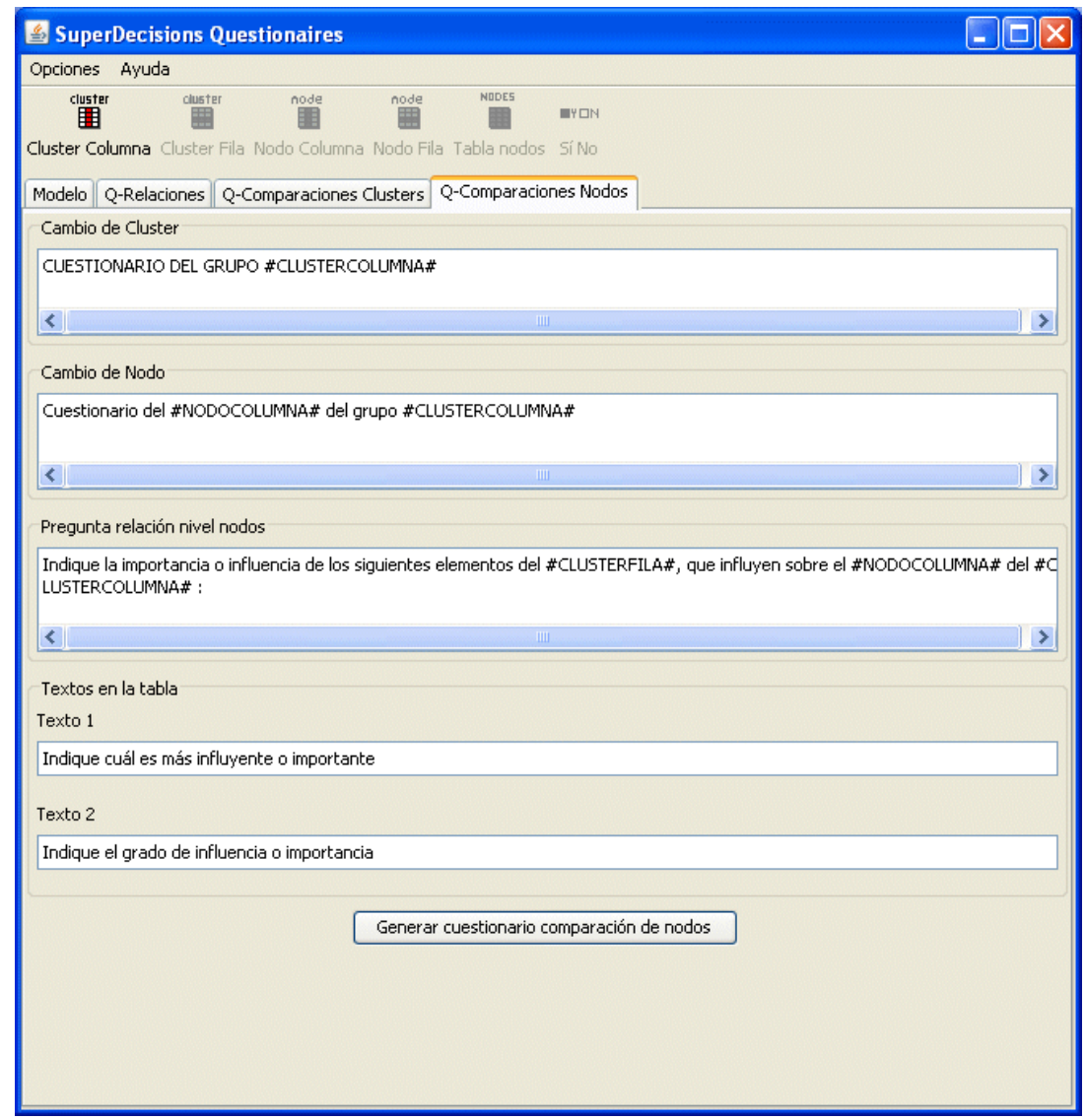

Figure 9.- Weighting nodes questionnaire

\section{Versions and available fund of the software}

One is working so that the user can choose between the English languages and Spanish for the software interface. In the moment to write this paper, the software is in tests phase, and it is expected that in the date of realization of ISAHP 2007, it will be available for its download in the web page of the Universidad Politécnica de Valencia group of multicriteria appraisal and decision (www.valoracionmulticriterio.upv.es) and in the web page of Departamento de Proyectos de Ingeniería (www.dpi.upv.es).

\section{Restrictions of the software}

The software generates the questionnaire in HTML format, so that later on the user can edit them if it considers it opportune. The software doesn't use the possible ratings that have been defined in the pattern ANP with SuperDecisions, to rate the alternatives. 


\section{Conclusions}

In this article a software tool are showed, that loads the Superdecisions files, and it elaborates automatically the questionnaires to define the relationships and to prioritize the existing relationships. This allows to speed up the obtaining of the opinions of the experts and to raise the data trace of the problem. 